\title{
Emprego de Endopróteses em Aorta Torácica: a Importância de Construir as Evidências
} Ver artigo relacionado
na página 46

\author{
Fabio B. Jatene ${ }^{1}$
}

0 emprego de endopróteses para tratamento das doenças da aorta vem crescendo de forma rápida e as próteses atualmente utilizadas, já em sua terceira geração, são muito mais adequadas que as inicialmente implantadas.

Inicialmente empregadas no tratamento de afecções da aorta abdominal, as endopróteses vêm sendo progressivamente mais utilizadas no segmento torácico. O grande entusiasmo com o tratamento endovascular das afecções da aorta deve-se ao caráter minimamente invasivo do procedimento, com menor agressão cirúrgica, por redução da perda sanguínea intraoperatória, e menor incidência de complicações neurológicas e renais, abreviando, assim, o período de internação hospitalar ${ }^{1-3}$. Entretanto, o uso das endopróteses não é isento de complicações, havendo em alguns casos necessidade de reintervenções, em decorrência principalmente da presença de endoleaks, migração da prótese ou, ainda, falha estrutural tardia.

O que tem chamado a atenção na literatura é a escassez de evidência científica que suporte a utilização das endopróteses. São poucos os trabalhos que conseguem comparar seu emprego em aorta torácica aos procedimentos cirúrgicos convencionais, pois a maioria das publicações é relacionada ao segmento abdominal. Revisão publicada pela The Cochrane Collaboration $^{4}$, em janeiro de 2009, confirmou a inexistência de estudos clínicos controlados e randomizados comparando os procedimentos endovascular e cirúrgico convencional no tratamento de aneurismas da aorta torácica.

A informação que se busca obter é se o tratamento endovascular consegue aliar pequena agressão a resultados superiores aos obtidos pelas técnicas classicamente empregadas. Dentre as várias questões sobre o tema, que necessitam de respostas, podemos desta- car duas: a primeira refere-se à comparação do emprego da endoprótese com tratamento cirúrgico convencional em aneurismas de arco aórtico e segmento descendente torácico; a segunda busca a comparação entre o tratamento clínico, conservador, para casos de dissecção aórtica tipo B não-complicada e o emprego de endoprótese.

Alguns trabalhos ${ }^{5,6}$ têm procurado respostas a essas questões. Dentre eles o trabalho de Alves et al. ${ }^{7}$, que compara o tratamento endovascular em pacientes portadores de afecções aneurismáticas e dissecção, publicado neste fascículo da Revista Brasileira de Cardiologia Invasiva. Nele, algumas observações chamam a atenção: há diferença entre o sucesso técnico e o sucesso aos 30 dias; o resultado do emprego das endopróteses em casos de dissecção aórtica é superior aos de aneurisma; e, por fim, em um período de seguimento inferior a 3 anos, $60 \%$ dos portadores de aneurisma e $43 \%$ com dissecção apresentaram falência tardia. É importante ressaltar que as próteses empregadas nesse estudo eram inferiores às atualmente utilizadas, pois eram mais rígidas e apresentavam menor navegabilidade.

Entretanto, são achados interessantes em um trabalho consistente e os resultados dos procedimentos atualmente realizados certamente serão superiores em decorrência da melhor seleção dos pacientes e do maior apuro técnico. O mais importante é a possibilidade de que trabalhos como esse possam fornecer dados comparativos e de casuística expressiva em um terreno muito pouco explorado, em que as investigações são escassas e indispensáveis para permitir a continuidade do método e seu aperfeiçoamento.

\section{CONFLITO DE INTERESSES}

O autor declarou inexistência de conflito de interesses.

\footnotetext{
1 Instituto do Coração do Hospital das Clínicas da Faculdade de Medicina da Universidade de São Paulo - São Paulo, SP, Brasil. Correspondência: Fabio B. Jatene. Instituto do Coração do Hospital das Clínicas da Faculdade de Medicina da Universidade de São Paulo. Av. Dr. Enéas de Carvalho Aguiar, 44 - 5o andar, bloco 2, sala 7 - Cerqueira César - São Paulo, SP, Brasil - CEP 05403-000 E-mail: fabiojatene@incor.usp.br 


\section{REFERÊNCIAS BIBLIOGRÁFICAS}

1. Svensson LG, Kouchoukos NT, Miller DC, Bavaria JE, Coselli JS, Curi MA, et al. Expert consensus document on the treatment of descending thoracic aortic disease using endovascular stent-grafts. Society of Thoracic Surgeons Endovascular Surgery Task Force. Ann Thorac Surg. 2008 Jan;85(1 Suppl):S1-41.

2. Zipfel B, Hammerschmidt R, Krabatsch T, Buz S, Weng Y, Hetzer R. Stent-grafting of the thoracic aorta by the cardiothoracic surgeon. Ann Thorac Surg. 2007;83(2):441-9.

3. Albuquerque LC, Braile DM, Palma JH, Saadi EK, Gomes WJ, Buffolo E. Diretrizes para o tratamento cirúrgico das doenças da aorta da Sociedade Brasileira de Cirurgia Cardiovascular. Rev Bras Cir Cardiovasc. 2007;22(2):137-59.

4. Abraha I, Romagnoli C, Montedori A, Cirocchi R. Thoracic stent graft versus surgery for thoracic aneurysm. Cochrane Database Syst Rev. 2009;(1):CD006796.

5. Nienaber CA, Zannetti S, Barbieri B, Kische S, Schareck W, Rehders TC. Investigation of stent grafts in patients with type B aortic dissection: design of the INSTEAD trial: a prospective, multicenter, European randomized trial. INSTEAD study collaborators. Am Heart J. 2005;149(4):592-9.

6. Eggebrecht $H$, Nienaber $C A$, Neuhäuser $M$, Baumgart D, Kische S, Schmermund A, et al. Endovascular stent-graft placement in aortic dissection: a meta-analysis. Eur Heart J. 2006;27(4):489-98.

7. Alves CMR, Fonseca JHP, Souza JAM, Kim HC, Esher G, Buffolo E. Tratamento endovascular nos aneurismas verdadeiros e na dissecção aórtica do tipo B: fase intra-hospitalar, seguimento de médio prazo e uma reflexão sobre seleção de pacientes. Rev Bras Cardiol Invas. 2009;17(1):46-51. 